\title{
La responsabilidad: un principio para retomar en la reflexión bioética
}

\author{
Juan Pablo Zapata-0spina ${ }^{1}$
}

\section{RESUMEN}

La ciencia y su brazo, la tecnología, pueden centrarse en una lógica antropocéntrica en la medida en que se empecinen en satisfacer únicamente las necesidades humanas a expensas del dominio (o destrucción) de la naturaleza y las especies coexistentes. También es posible que se pongan al servicio de poderes económicos y políticos y se investigue únicamente bajo una lógica centrada en la ganancia. En medio de este panorama, se hace un llamado a un acto de conciencia para retomar la propuesta del filósofo alemán Hans Jonas sobre el principio de responsabilidad, según el cual, es necesaria una reflexión más allá de las relaciones inmediatas, que incluya a la naturaleza, los animales y las generaciones futuras en la formulación de una nueva ética que debe enseñarse y practicarse desde la academia.

\section{PALABRAS CLAVE}

Educación; Ética Basada en Principios; Ética en Investigación; Responsabilidad Social; Revisión Ética

\section{SUMMARY}

\section{Responsibility: a principle to resume in bioethical reflection}

Science and its arm, technology, may focus on an anthropocentric logic if they insist on satisfying only human needs at the expense of the mastery (or destruction) of nature and

\footnotetext{
Médico y cirujano, especialista en Psiquiatría, magíster en Epidemiología Clínica. Profesor. Instituto de Investigaciones Médicas. Facultad de Medicina. Universidad de Antioquia. Medellín, Colombia.

Correspondencia: Juan Pablo Zapata 0spina; juanp.zapata@udea.edu.co

Recibido: enero 21 de 2019

Aceptado: mayo 21 de 2019
}

Cómo citar: Zapata-Ospina JP. La responsabilidad: un principio para retomar en la reflexión bioética. latreia. 2019 0ct-Dic;32(4):338-345. D0I 10.17533/udea.iatreia.30. 
coexisting species. It is also possible that they serve to economic and political powers and investigate only under a logic focused on profit. Within this panorama, a call is made to an act of conscience for the proposal of the German philosopher Hans Jonas on responsibility principle, which claims for a reflection beyond the immediate relations, including nature, animals and future generations in the formulation of a new ethics that should be taught and practiced from the academy.

\section{KEYWORDS}

Education; Ethics, Research; Ethical Review; Principle-Based Ethics; Social Responsibility

\section{INTRODUCCIÓN}

\begin{abstract}
"Actúa de tal modo que Ios efectos de tu acción sean compatibles con la permanencia de una vida humana auténtica»
\end{abstract}

Hans Jonas

La búsqueda constante para satisfacer las propias necesidades ha sido el motor de la supervivencia del hombre. En esa búsqueda, y con la inherente curiosidad de quien observa, se ha venido forjando el conocimiento sobre la naturaleza y las leyes que la gobiernan. Por medio de la investigación se recogen y sistematizan observaciones para construir teorías para entender el mundo, lo que permite llegar a manipularlo y transformarlo ${ }^{(1)}$. En ese punto surgen nuevas necesidades que dan origen al proceso iterativo que, en términos críticos, es la ciencia. De ella se han desprendido grandes beneficios: pasar de las herramientas rudimentarias de la Edad de Piedra a la domesticación de plantas y animales y la manipulación de metales, hasta llegar a desarrollar sociedades que transmiten información a cualquier lugar en la Era de las tecnologías de la información y la comunicación. Ese es, en parte, el mecanismo que nos ha conducido a la evolución que hemos tenido como especie ${ }^{(2)}$.

En el ámbito del cuidado de la salud se han expandido los soportes mismos de la existencia humana con un incremento de la expectativa de vida. EI avance en procedimientos diagnósticos, nuevos medicamentos y tecnologías, el descubrimiento del genoma y su manipulación han revolucionado el mundo médico y han puesto nuevos estándares del proceso saludenfermedad ${ }^{(3)}$. No obstante, estas acciones no están libres de generar efectos negativos que repercuten en el mundo que intentamos comprender y del cual la especie humana hace parte. Es probable que lo que surge como solución a una necesidad inmediata no sea dimensionado en sus consecuencias póstumas $y$ pueda representar efectos deletéreos. En esa medida es necesario reflexionar y proponer soluciones para lidiar con las consecuencias no deseadas. A medida que se profundiza en lo que conocemos y manipulamos, Ios cuestionamientos que debemos hacernos deben ampliarse para asegurar la vida misma, que es en últimas, el sentido de la bioética ${ }^{(4)}$.

En este artículo se esbozan algunos riesgos del desarrollo científico y tecnológico en el marco de la investigación en salud. Se plantea la incorporación del principio de responsabilidad a la reflexión bioética con miras a la búsqueda de soluciones para aminorar y prevenir los daños.

\section{LA DOMINANCIA DE UNA ESPECIE}

A través de la ciencia y la tecnología el hombre busca conocer la realidad que lo rodea $y$ aplicar dicho conocimiento de tal manera que pueda mejorar sus condiciones de vida con la invención y producción de bienes materiales y culturales ${ }^{(5)}$. No obstante, este escenario puede resultar el asidero de una lógica antropocéntrica, si se empecina en llegar a dominar la naturaleza con la intencionada falta de reconocimiento de las implicaciones de cada creación y solo se concentra en satisfacer una necesidad humana ${ }^{(6)}$.

Ninguna otra especie en el planeta ha tenido una dominancia tan extrema sobre el medio ambiente $y$ las otras especies coexistentes como la humana. Por ejemplo, la concentración de metano y óxido nitroso, los gases que más contribuyen al efecto invernadero, se ha incrementado ostensiblemente después de la Revolución Industrial ${ }^{(7,8)}$. La producción de alimentos es una de las principales fuentes en la emisión de este tipo de gases y, dado el aumento en la población mundial, se intensificará la emisión de dichos gases, lo que puede amenazar la vida ${ }^{(9)}$. En la producción y 
el uso de productos farmacéuticos para la atención de enfermedades humanas se generan peligros. Se han encontrado, por ejemplo, antibióticos en agua para el consumo y en aguas dulces corrientes, que generan efectos tóxicos en invertebrados; estrógenos en ambientes acuáticos, que pueden llegar a afectar la reproducción y el desarrollo de anfibios; y agentes citotóxicos en el agua de hospitales que no siempre alcanzan a eliminarse en las plantas de tratamiento de aguas ${ }^{(10)}$.

En Colombia la situación de los ecosistemas es crítica pues se estima que más del $90 \%$ de los municipios tienen problemas de agua, bien sea por la calidad o por la disponibilidad. El deterioro en la calidad es evidenciado por la presencia de bacterias coliformes en acuíferos, el vertimiento de aguas residuales que contienen químicos y sólidos provenientes de la industria y los hogares, así como materia orogánica y plaguicidas derivados de actividades agrícolas ${ }^{(11)}$.

Es un hecho que la experimentación con animales en la investigación biomédica básica y clínica ha llevado a avances en el campo de la salud humana. Desde Galeno (131-201 a.C.) y Vesalius (1514-1564), el conocimiento sobre anatomía y fisiología se produjo en gran parte a partir de vivisecciones ${ }^{(12)}$. Claude Bernard, padre de la medicina experimental, las tomaba como "totalmente concluyentes para la toxicología y la higiene del hombre" (13). Ejemplos más recientes han sido publicados por la Asociación Americana del Corazón, donde investigadores indujeron falla cardiaca a perros implantándoles un marcapasos y llevándolos a taquicardia por un mes; la falla cardiaca se confirmó por síntomas como letargia, anorexia, disnea y ascitis; de modo que, a partir de este punto, un grupo fuera tratado con terapia de resincronización y otro siouiera como control. Del examen anatomopatológico deI corazón se pudieron entender aspectos de la mecánica del corazón, la biología de los cardiomiocitos y el beneficio —en lo molecular- de la resincronización ${ }^{(14,15)}$. Al igual que en otros ejemplos, no siempre es posible encontrar un razonamiento sobre los riesgos y beneficios en los animales ni un esfuerzo por cuidarlos de manera diogna; es decir que, bajo el escrutinio bioético, estos experimentos no tendrían una justificación o al menos serían susceptibles de reformas ${ }^{(16)}$.

La relación del hombre con el medio ambiente y las demás especies ha planteado problemas éticos que no se habían vislumbrado antes, porque no se tenía conocimiento de los daños y porque la reflexión se centraba en las relaciones entre seres humanos. A partir de la década de 1970 estos problemas fueron visibilizados gracias a la movilización de entes no gubernamentales y algunos Estados, lo que redundó en acuerdos para la protección del medio ambiente. Por ejemplo, el Informe Bruntland de la Oroganización de Naciones Unidas (ONU) planteó una postura sobre el desarrollo sostenible y las restricciones ecológicas necesarias para asegurar la satisfacción de las necesidades en el futuro ${ }^{(17)}$.

Al señalar la crisis ecológica se fueron sentando las bases de un discurso sobre el respeto por la tierra, sus ecosistemas y el otro no-humano, que correspondería a una ecoética ${ }^{(18)}$. La conformación de este nuevo y más amplio nivel de reflexión ha sido llamada también como macroética, una ética del humano como especie que no puede disociarse de su contexto cultural, económico y político, pero tampoco del contexto que es el mundo y la naturaleza. Sería la reflexión de la vida misma a gran escala ${ }^{(19)}$.

\section{EL LUCRO COMO EL OBJETIVO DE LA CIENCIA}

Los productos científicos no pueden verse de manera diacrónica puesto que tienen una caroga histórica. Cuando se produce un descubrimiento o se desarrolla una técnica, existe un interés específico en que cumpla una función en ese momento de la historia. Así lo científico sigue un fin histórico determinado por el grupo social, lo que se ha entendido como la politización de Ia tecnociencia ${ }^{(20)}$. Esto también implica que los avances puedan ponerse al servicio de intereses de algunas personas o grupos impregnados de mezquindad y lucro desenfrenado. En este marco la ciencia se convertiría en una fuente de riqueza y poder, estaría subordinada a una Iógica centrada en Ia ganancia.

Algunas investigaciones y desarrollos de tecnologías están al servicio de organizaciones que detentan poder económico y político. Sabemos, por ejemplo, que los conocimientos sobre la fisión nuclear condujeron al desarrollo de la bomba atómica que arrasó con Hiroshima y Nagasaki durante la Segunda Guerra Mundial ${ }^{(21)}$, que el avance en la medicina con la ampliación de los detalles anatómicos del cuerpo humano fue 
perpetrada por los Nazi en el Holocausto (22), que desde entonces se han financiado investigaciones que buscan soluciones para el desarrollo armamentista para mantener la "seguridad colectiva" de algunos países ${ }^{(6)}$.

Más recientemente hemos visto que desde la investigación biomédica se ha favorecido a las compañías farmacéuticas y sus productos. Los errores sistemáticos en ensayos clínicos con una incorrecta elección de comparadores o la falta de publicación de resultados negativos es un claro ejemplo de ello ${ }^{(23)}$. El patrocinio de las industrias de alimentos ha llevado a que la literatura científica que leemos en nutrición esté plagada de conclusiones favorables hacia los productos que promocionan ${ }^{(24)}$.

Así las cosas, la promesa de solucionar problemas o remediar necesidades llega a convertirse en una estrategia buscadora de lucro y no de fines más trascendentales como el bien común. Se convierte en una ciencia injusta al representar un bien particular en lugar del común. Estaríamos hablando de una concepción de justicia en un sentido distributivo que rige las relaciones entre los miembros de una sociedad: quienes ostentan poder y los ciudadanos rasos (25). La repartición de bienes en el caso de la ciencia y sus productos debería darse de manera proporcional para que los más pobres accedieran a ellos por la vía del bien común y el bienestar colectivo, que deberían encarnar quienes tienen más poder y riqueza.

Es necesario advertir también que esta lógica nos puede atrapar en el espejismo de que una nueva invención resuelve un problema o una necesidad, cuando en realidad es porque una invención se ha vuelto obsoleta o no representa lucro. Por ejemplo, la empresa farmacéutica Lundbeck comercializaba el antidepresivo citalopram, pero cuando su patente iba a caducar, lanzó únicamente la parte activa de la misma molécula bajo el nombre de escitalopram, con una campaña en la que afirmaba que era más efectivo que su predecesor para tratar la depresión a pesar de que ambos medicamentos tienen el mismo principio activo. Se determinó que la empresa había infringido el código de práctica de la industria del Reino Unido en la forma en que anunció el escitalopram ${ }^{(26)}$. De modo que la comercialización de un "nuevo antidepresivo" no obedecía a un interés de mejorar la vida de las personas con este trastorno sino, por el contrario, de aliviar las ganancias de una empresa.
Un problema adicional del crecimiento tecnocientífico es el debilitamiento de la esencia humana. Las revoluciones científicas se están haciendo cada vez más cortas y no se acompañan necesariamente de un desarrollo humano ${ }^{(19)}$. La ciencia deja de ser la exploración que hace el hombre y se convertiría en una especie de agente autónomo, un poder que modifica todo a su antojo, incluso lo que se concibe como hombre. La especialización cada vez más minúscula de las ciencias lo mutilaría, lo borraría como unidad. Esta hipérbole señala una deshumanización en la medida en que nos convertimos en objetos de investigación y dejamos de ser los sujetos que investigan; somos dependientes de los objetos tecnológicos, buscamos la manipulación de nosotros mismos con las tecnologías o anulamos nuestra racionalidad y la sometemos a las exigencias tecnológicas ${ }^{(27,28)}$.

\section{EL PRINCIPIO DE LA RESPONSABILIDAD COMO OPORTUNIDAD}

En medio de estas adversidades es posible darse la oportunidad de pensar y pensarse diferente. La proximidad de la muerte termina por hacer consciente la vida y convierte a la agonía en una oportunidad $y$ en este sentido la muerte puede llenar de significado la vida. En nuestro caso hemos tenido grandes catástrofes y acumulación de daños que pueden verse como una desintegración, entendiendo que el mundo mismo puede ser un organismo en agonía ${ }^{(29)}$. Sin embargo, de ahí puede surgir un acto de conciencia, una apuesta por la reintegración. Ver de cerca el daño por las atrocidades perpetuadas en la Segunda Guerra Mundial, por ejemplo, condujo al planteamiento de preguntas éticas sobre el desarrollo científico que fueron plasmadas en el Códiogo de Nuremberg (1948) o la Declaración de los Derechos Humanos de la ONU (1948), origen del marco ético y jurídico actual ${ }^{(30)}$.

En este proceso de muerte por daño real y potencial de los desarrollos tecnológicos y el desconocimiento de sus consecuencias, resulta conveniente retomar la propuesta del filósofo alemán Hans Jonas de una moderación responsable y la formulación de una nueva ética que reflexione más allá de las relaciones inmediatas e incluya a la naturaleza y a las generaciones futuras ${ }^{(31)}$. En su libro EI principio de responsabilidad ${ }^{(32)}$, plantea 
Ia necesidad de asumir consecuencias, es decir, ser responsable: ¿qué le sucederá a eso si no me ocupo de ello? La propuesta de Hans Jonas de una ética Ilamada a superar el antropocentrismo queda reflejada en la frase "no pongas en peligro la continuidad indefinida de la humanidad en la tierra” (32). Las dimensiones de esta responsabilidad implican, de un lado, la desaparición del criterio de proximidad espacial y temporal para evaluar la importancia de una acción, y de otro, la introducción de un factor de acumulación en el sentido de que las acciones y sus efectos se suman. Esto implica un conocimiento predictivo que, paradójicamente, depende del saber tecnocientífico. La diferencia está en la intencionalidad que reviste ese conocimiento, pues se dirige más a una especie de autovigilancia que a la búsqueda de poder. Esta posición fue un avance en el camino deontológico que ya había iniciado Kant, quien anteponía el deber u obligación a las preferencias y abogaba por un razonamiento a priori para llevar a la realidad un ideal, aunque con un imperativo categórico más individualista que no contenía el futuro ${ }^{(33)}$.

Aunque se promueva la superación de la lógica antropocéntrica, es preciso reflexionar sobre los alcances que tiene la ciencia sobre las condiciones humanas. Jonas se interesó por los adelantos tecnológicos que podrían prolongar la vida al punto distópico de no tener que morir ${ }^{(32)}$. Habría que pensar hasta qué punto es justo aumentar la esperanza de vida a expensas de solo ganar la batalla contra la muerte, envejecer en condiciones indignas, quitarles oportunidades a los jóvenes, etc. Las reglas de la vida en la tierra, que parecían inquebrantables, pueden modificarse sin que lleguemos a conocer del todo sus consecuencias, pero hay que pensarlas. Algunos han planteado que, aunque tener vida pueda ser intrínsecamente valioso, no sería justo pensar en su prolongación cuando existen países subdesarrollados cuya expectativa de vida no excede los 40 años, sería entonces un lujo para quien pueda pagarlo ${ }^{(34)}$. O no sería conveniente porque se alteraría el significado mismo de la vida al punto de perder el sentido de trascendencia ${ }^{(35)}$. Cada argumento que pueda darse en el tema puede controvertirse, pero el punto es la visión responsable de una acción, la que busca más allá de las consecuencias inmediatas.

Igualmente, Jonas se refirió al tema de la manipulación genética y el descubrimiento del genoma. Para él, se trata de una maniobra con la que el hombre pretende apoderarse de su propia evolución, no solo para conservar la especie, sino para diseñar e introducir mejoras ${ }^{(32)}$. Esto plantea las cuestiones sobre quién define la mejora y para qué las utilizaría. Hoy en día existen modelos predictivos que utilizan variantes genéticas asociadas a enfermedades con el fin de determinar la probabilidad de que se presenten. Aunque su capacidad predictiva no ha sido correctamente evaluada $y$ requiere que se haga una validación en cada población, estos modelos poligénicos se han venido comercializando sin ningún tipo de restricción a cualquier consumidor y bajo pautas publicitarias falsas ${ }^{(36)}$. EI propósito que se pretende alcanzar con estas pruebas no siempre resulta claro, lo cual debería ser la prioridad en este tipo de investigaciones ${ }^{(37)}$. Su aplicación clínica en masa no debería promulgarse aún, ya que ameritaría una mayor investigación ${ }^{(38)}$, hecho que demuestra el interés comercial que hay detrás de ellos.

Además del futuro de la humanidad, el principio de responsabilidad se centra en la naturaleza, la vida de la Tierra y todo lo que habita en ella. Ya se han señalado los daños producidos, que serían por sí solos argumento para su protección. Aquí podría verse a la responsabilidad como cercana a la sensación de remordimiento ${ }^{(31)}$. Pero debería existir una solidaridad con la naturaleza dado que es nuestra morada, con su propia dignidad y esencia, por lo que, en función de nuestra capacidad de comprenderla, tendríamos la obligación de protegerla ${ }^{(32)}$. Algunos han objetado que en este planteamiento hay una lógica antropocéntrica en la medida en que la naturaleza se cuida para beneficio del hombre; sin embargo, además de la solidaridad, por su capacidad de intervención y razonamiento, Jonas argumentó que "el hombre es el único ser conocido que puede tener responsabilidad, por ello, justamente, la tiene" (39). Se puede resumir, entonces, en que son el poder y la razón las que conducen a la responsabilidad.

\section{LA RESPONSABILIDAD PUESTA EN PRÁCTICA}

La bioética es un ejercicio que involucra al mismo tiempo una actividad cognitiva, una experiencia $y$ un aspecto normativo dentro de un contexto particular en el marco de las bases biológicas de la existencia humana ${ }^{(4)}$. Involucrar a este ejercicio principios como el de la responsabilidad es lo que algunos han 
Ilamado como la recuperación de la virtud en bioéti-

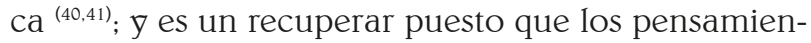
tos sobre alcanzar perfección y plenitud ya ocupaban las mentes de los filósofos clásicos. Aquí se enfatiza sobre la experiencia del hombre, su motivación y su disposición para obrar, pues en el acto manifiesta y potencia las propias cualidades.

Esta reflexión no es un llamado a abandonar la ciencia o que la humanidad deba condenarse al oscurantismo. Tampoco se pretende desconocer el gran impacto positivo que han tenido algunos avances científicos. La tecnociencia como objeto no es en sí misma buena o mala, el juicio de valor se debería hacer sobre el uso que el ser humano como sujeto le ha dado ${ }^{(27)}$. Por ello se trata más bien de seguir haciendo ciencia, pero con conciencia, de impedir el desarrollo incontrolado que perjudique el futuro del hombre. Es desarrollar una reflexión intencionada que oriente la forma de hacer ciencia; que no se limite solo a reglamentar el uso de las tecnologías, sino que argumente la protección de la vida humana y la naturaleza a través de ellas. Que sea esa la entelequia de todas las formas de hacer ciencia.

Con rl movimiento por una Conducta Responsable en Investigación, que ya ha tenido eco en Estados Unidos, se debe hacer explícito el compromiso por practicar y divulgar las aspiraciones de una ciencia honesta, íntegra y dirigida a intereses más altruistas. Debe incluir a la investigación con animales, en seres humanos, el trabajo científico colaborativo, el proceso de revisión por pares, las prácticas de publicación y autoría, el abordaje de los conflictos de interés y las malas prácticas científicas y la capacitación continua ${ }^{(16)}$.

Los comités de ética de la investigación en seres humanos y animales están llamados a participar en este proceso. Deben verse como un espacio democrático donde confluyan los intereses del ciudadano de a pie y del investigador, además de que se puedan formar consensos pragmáticos tras la deliberación exhaustiva ${ }^{(42)}$. Deben generarse encuentros para enseñar y compartir las discusiones que sean producto de las evaluaciones y seguimientos de proyectos.

Los investigadores también deben ser vistos como una población vulnerable en la investigación, pues pueden caer en algunas situaciones inconvenientes por falta de conocimientos puntuales, estrés, presión por la publicación de manuscritos, entre otros ${ }^{(43,44)}$.
La idea es que, a partir de la identificación de las necesidades humanas y del entorno, el investigador elabore preguntas relevantes para llevar a cabo sus investigaciones. Esto implica que se sustente en un modelo teórico adecuado y se puedan predecir los beneficios que traerá la realización de la investigación, así como los riesgos que puede implicar.

Mantener la propuesta de la responsabilidad en el campo de la acción también es posible si nos enfocamos en la educación, si tomamos espacios intencionados de reflexión en los diferentes ámbitos académicos. Una educación que obviamente no esté filtrada por esos intereses, muchas veces sutilmente ${ }^{(45)}$, sino que sea independiente y trasparente. Es necesaria la inclusión transversal en los currículos de los programas educativos de materias sobre la naturaleza de las ciencias y sostenibilidad ambiental ${ }^{(46,47)}$, así como el entrenamiento formal y práctico en bioética ${ }^{(48)}$. Es la reflexión la que puede transformar las formas de actuar. Es actuando que se generan tradiciones, por lo que los profesores e investigadores también estamos llamados al entrenamiento teórico continuo y a la puesta en práctica en el quehacer.

\section{A MANERA DE CONCLUSIÓN}

El llamado a una reflexión bioética no se restringe al grupo específico de quienes hacen ciencia, sino que se dirige a todos los estamentos sociales y usuarios de los hallazgos científicos, es un compromiso de todos como ciudadanos del mundo. El momento de reflexionar y actuar es ahora, pues siguiendo la lógica de Jonas, es lo más responsable que podemos hacer. De no hacerlo, es posible que nuestra supervivencia actual y futura se vea amenazada. Es el futuro el que está en juego.

\section{CONFLICTOS DE INTERESES}

Ninguno por declarar.

\section{REFERENCIAS BIBLOGRÁFICAS}

1. Lolas F, Quezada A, Rodríguez E. Investigación en salud. Dimensión ética. Acta bioeth. 2006;12(2):257-8. 
2. Schick K, Toth N. The origin of the Genie. En: Lightman A, Sarewitz D, Desser C, editores. Living with the Genie: essays on technology and the quest for human mastery. Washington, DC: Island Press; 2003. p. 23-34.

3. Suazo M. La bioética en el desarrollo científico tecnológico del área de la salud: enfermería y ética del cuidado. Cienc Soc. 2001;XXVI(1):7-15.

4. Ladrière J. Del sentido de la bioética. Acta Bioeth. 2000; VI(2): 197-218.

5. Bunge M. Ciencia y Desarrollo. Buenos Aires: Ediciones Siglo Veinte; 1980. 176 p.

6. Willmott H. Science, governance and self-understanding: from anthropocentricism to ecocentrism? Crit policy Stud. 2014;8(1):22-40.

7. Stern DI, Kaufmann RK. Estimates of global anthropogenic methane emissions 1860-1993. Chemosphere. 1996;33(1):159-76.

8. Davidson E. The contribution of manure and fertilizer nitrogen to atmospheric nitrous oxide since 1860 . Nat Geosci. 2009;2:659-62.

9. Hedenus F, Wirsenius S, Johansson DJA. The importance of reduced meat and dairy consumption for meeting stringent climate change targets. Clim Change. 2014;124(1-2):79-91.

10. Deblonde T, Hartemann P. Environmental impact of medical prescriptions: assessing the risks and hazards of persistence, bioaccumulation and toxicity of pharmaceuticals. Public Health. 2013;127(4):312-7.

11. Liévano-León A. La bioética y el uso de los ecosistemas acuáticos. En: Escobar Triana J, editor. Memorias de la mesa sobre Bioética Ambiental. Bogotá D.C.; 2010. p. 119-22.

12. Miziara ID, Magalhães AT de $M$, Santos $M$ d'Aparecida, Gomes EF, Oliveira RA. Research ethics in animal models. Braz J Otorhinolaryngol. abril de 2012;78(2):128-31.

13. LaFollette H, Shanks N. Animal experimentation: The legacy of Claude Bernard. Int Stud Philos Sci. el 9 de enero de 1994;8(3):195-210.

14. Kääb S, Nuss HB, Chiamvimonvat N, O’Rourke B, Pak $\mathrm{PH}$, Kass DA, et al. Ionic mechanism of action potential prolongation in ventricular myocytes from dogs with pacing-induced heart failure. Circ Res. febrero de 1996;78(2):262-73.
15. Chakir K, Daya SK, Tunin RS, Helm RH, Byrne MJ, Dimaano VL, et al. Reversal of Global Apoptosis and Regional Stress Kinase Activation by Cardiac Resynchronization. Circulation. 2008;117(11):1369-77.

16. Ferdowsian HR, Gluck JP. The Ethical Challenges of Animal Research. Cambridge Q Healthc Ethics. 2015;24(4):391-406.

17. Amaro Cano M deI C. La invasión tecnológica en las ciencias médicas y su repercusión ética en el desarrollo sostenible. Cuad Bioética. 2000;2:200-9.

18. Gómez Giraldo LJ. De la bioética a la ecoética. Gest Ambient. 2015;18(1):147-57.

19. Morán AA. La ética de la información y la infoesfera. Escritos. 2013;21(45):21-37.

20. Hottois G. EI paradiogma bioético: Una ética para la tecnociencia. Barcelona: Editorial Anthropos; 1991. 208 p.

21. Loh S-L. Beyond Peace: Pluralizing Japan's Nuclear History. Asia-Pacific J. 2013;10(11 (6)):1-2.

22. Lefor AT. Scientific misconduct and unethical human experimentation: historic parallels and moral implications. Nutrition. 2005;21(7-8):878-82.

23. Lexchin J. Pharmaceutical industry sponsorship and research outcome and quality: systematic review. BMJ. 2003;326(7400):1167-70.

24. Lesser LI, Ebbeling CB, Goozner M, Wypij D, Ludwig DS. Relationship between Funding Source and Conclusion among Nutrition-Related Scientific Articles. PLoS Med. 2007;4(1):e5.

25. Gracia D. Profesión médica, investigación y justicia sanitaria. Bogotá D.C: Editorial EI Búho; 2002. 152-176 p.

26. Walsh K. The winner takes it all. J Med Ethics. 2005;31(5):267-267.

27. Aguilar Gordón F. Reflexiones filosóficas sobre la tecnología y sus nuevos escenarios. Sophía. 2011;1(11):123.

28. Giraldo FL. La naturaleza humana ante el desarrollo científico y tecnológico. Trilogia. 2011;4:115-27.

29. Van Hooft S. What is death? En: LIFE, DEATH, AND SUBJECTIVITY Moral Sources in Bioethics. Amsterdam: Rodopi; 2004. p. 143-52.

30. Zuleta Salas GL. La aparición de la bioética y el porqué de la misma. Rev Lasallista Investig. 2014;11(1):23-34.

31. De Siqueira JE. EI principio de responsabilidad de Hans Jonas. Acta Bioeth. 2001; VII(277-285). 
32. Jonas H. El principio de responsabilidad. Ensayo de una ética para la civilización tecnológica. Barcelona: Herder Editorial; 1995. 400 p.

33. Restrepo Tamayo JC. La teoría de la responsabilidad como imperativo ético. Hans Jonas y el principio axiológico para la tecnociencia. Escritos. 2011;19(42):79-121.

34. Davis JK. Collective Suttee: Is It Unjust to Develop Life Extension if It Will Not Be Possible to Provide It to Everyone? Ann N Y Acad Sci. 2004;1019(1):535-41.

35. Pijnenburg MAM, Leget $C$. Who wants to live forever? Three arguments against extending the human lifespan. J Med Ethics. 2007;33(10):585-7.

36. Kalf RR, Bakker R, Janssens ACJ. Predictive ability of direct-to-consumer pharmacogenetic testing: when is lack of evidence really lack of evidence? Pharmacogenomics. 2013;14(4):341-4.

37. Martens FK, Janssens ACJW. How the Intended Use of Polygenic Risk Scores Guides the Design and Evaluation of Prediction Studies. Curr Epidemiol Reports. 2019;6(2):184-90.

38. Chasioti D, Yan J, Nho K, Saykin AJ. Progress in Polygenic Composite Scores in Alzheimer's and Other Complex Diseases. Trends Genet. 2019;35(5):371-82.

39. Rosales A. Naturaleza orgánica y responsabilidad ética: Hans Jonas y sus críticos. Trans/Form/Ação (São paulo). 2004;27(2):97-111.
40. Garcés LF. En la búsqueda de un modelo bioético para la experimentación con animales: elementos para su fundamentación. Prod + Limpia. 2014;9(1):124-40.

41. Díaz-García A. La ética de la virtud y la bioética. Rev Colomb Psiquiatr. 2009;4(1):93-128.

42. Carrasco N. Gilbert Hottois y los comités de ética: ¿una apuesta insostenible? Rev Laguna. 2010;27:57-69.

43. Amos L. Researcher vulnerability: An overlooked issue in vulnerability discourses. Sci Res Essays. 2014;9(16):737-43.

44. Sherry E. The vulnerable researcher: facing the challenges of sensitive research. Qual Res J. 2013;13(3):278-88.

45. Moynihan R. Doctors' education: the invisible influence of drug company sponsorship. BMJ. 2008;336(7641):416-7.

46. Blackley S, Sheffield R. Environment: Re-negotiating the $\mathrm{E}$ in STEM Education. Eco-Thinking. 2016;1.

47. Rudolph JL. Reconsidering the "nature of science" as a curriculum component. J Curric Stud. 2000;32(3):403-19.

48. Greenberg RA, Kim C, Stolte H, Hellmann J, Shaul RZ, Valani R, et al. Developing a bioethics curriculum for medical students from divergent geo-political regions. BMC Med Educ. 2016;16(1):193. 